PROCEEDINGS OF THE

AMERICAN MATHEMATICAL SOCIETY

Volume 140, Number 12, December 2012, Pages 4235-4242

S 0002-9939(2012)11290-0

Article electronically published on April 17, 2012

\title{
QUASI-WANDERING SUBSPACES IN A CLASS OF REPRODUCING ANALYTIC HILBERT SPACES
}

\author{
YONG CHEN \\ (Communicated by Richard Rochberg)
}

Abstract. We prove that a class of reproducing analytic Hilbert spaces with $\mathcal{U}$-invariant kernels on the unit ball has the quasi-wandering property for the $d$-shift operator tuple.

\section{INTRODUCTION}

For a given operator $T$ on a separable Hilbert space $H$, a closed subspace $M$ of $H$ is called an invariant subspace for $T$ if $T M \subset M$. For an invariant subspace $M$ of $H$ for $T$, the space $M \ominus T M$ is called the wandering subspace and $P_{M} T M^{\perp}$ the quasiwandering subspace respectively for $M$, where and in what follows $P_{M}$ denotes the projection onto a closed subspace $M$ and $M^{\perp}=H \ominus M, T M^{\perp}=\left\{T x: x \in M^{\perp}\right\}$.

For a subset $E$ of $H$, we shall denote by $[E]_{T}$ the smallest invariant subspace of $H$ for $T$ containing $E$. In other words, $[E]_{T}$ is the norm-closed linear span of functions of the form $T^{k} \psi$ for $\psi \in E$ and $k=0,1, \cdots$.

An operator $T$ on a Hilbert space $H$ has the wandering property (briefly, the W-property) if for each nontrivial invariant subspace $M$ of $H$ for $T, M=[M \ominus$ $T M]_{T}$ and has the quasi-wandering property (briefly, the QW-property) if $M=$ $\left[P_{M} T M^{\perp}\right]_{T}$.

Let $\mathbb{D}$ be the open unit disk in the complex plane $\mathbb{C}$ and $H$ denote a reproducing kernel Hilbert space of analytic functions on $\mathbb{D}$. There are many examples where the shift operator $S$, defined by

$$
(S f)(z)=z f(z), \quad f \in H, z \in \mathbb{D},
$$

has the W-property. In $\mathrm{Beu}$, Beurling proved that if $M \neq 0$ is an invariant subspace of the Hardy space $H^{2}(\mathbb{D})$, then $M \ominus S M$ is a one-dimensional subspace spanned by an inner function $\eta$ and

$$
M=[\eta]_{S}=[M \ominus S M]_{S} .
$$

Beurling's theorem has played an important role in operator theory, function theory and their intersection, function-theoretic operator theory. However, despite the great development in these fields over the past fifty years, it is only recently that progress has been made in proving analogues for the other classical Hilbert spaces,

Received by the editors February 7, 2011 and, in revised form, May 28, 2011.

2010 Mathematics Subject Classification. Primary 47B37; Secondary 46E22.

Key words and phrases. Invariant subspace, quasi-wandering subspace, $d$-shift operator tuple, reproducing analytic Hilbert space.

The author was supported in part by NNSFC (Nos. 10971195 and 11001246), ZJNU (Nos. Y6110260 and Y6100007), and Tianyuan FC (No. 11126259). 
the Dirichlet space and the Bergman space. In Ric, Richter proved that the analogue of Beurling's theorem is true in the Dirichlet space $\mathcal{D}$. It is well known that the invariant subspace lattice of the Bergman space $L_{a}^{2}(\mathbb{D})$ is very complicated. In fact, the dimension of the wandering subspace $M \ominus S M$ can be an arbitrary positive integer or $\infty$ ( $[\mathrm{Hed}]$ ). However, a big breakthrough in the study of the analogue of Beurling's theorem on the Bergman space was made by A. Aleman, S. Richter and C. Sundberg ( ARS $)$. They proved that any invariant subspace $M$ of the Bergman space $L^{2}(\mathbb{D})$ also has the W-property.

The quasi-wandering subspace was studied recently by Izuchi in [zu]. Let $M$ be a nontrivial invariant subspace of $H^{2}(\mathbb{D})$ for $S$. Since in this case $S$ is an isometry, i.e., $S^{*} S=1$, then one easily sees that $P_{M} S M^{\perp} \subset M \ominus S M$. On the other hand, it is easy to check (see [zu]) that

$$
\operatorname{dim} M \ominus S M \leq \operatorname{dim} P_{M} S M^{\perp} .
$$

Thus we have

$$
P_{M} S M^{\perp}=M \ominus S M .
$$

So in the one-variable Hardy space case, a quasi-wandering subspace coincides with a wandering subspace for $M$. It is not the case in the Bergman space although (1.1) still holds. However, in [Izu it is shown that the shift operator on the Bergman space $L_{a}^{2}(\mathbb{D})$ has the QW-property. This result is seen as a counterpart to the theorem of Aleman-Richter-Sundberg.

We say that a closed subspace $M$ of Hilbert space $H$ is invariant for a $d$-tuple of operators $\bar{T}=\left(T_{1}, \ldots, T_{d}\right)$, if $T_{i} M \subset M$ for all $i=1, \ldots, d$. Set

$$
P_{M} \bar{T} M^{\perp}=P_{M} T_{1} M^{\perp}+\cdots+P_{M} T_{d} M^{\perp} .
$$

We say that an operator tuple $\bar{T}=\left(T_{1}, \ldots, T_{d}\right)$ has the QW-property if for each nontrivial invariant subspace $M$ of $H$ for $\bar{T}, M=\left[P_{M} \bar{T} M^{\perp}\right]_{\bar{T}}$.

Motivated by the work of [Izu, in this paper we will consider the QW-property for a $d$-tuple of operators $\bar{S}=\left(S_{1}, \ldots, S_{d}\right)$, called $d$-shift, on a reproducing analytic Hilbert space $H_{d}^{k}$ on the unit ball $\mathbb{B}_{d}$ of $\mathbb{C}^{d}$ defined by a $\mathcal{U}$-invariant kernel $k$. Here

$$
\left(S_{i} f\right)(z)=z_{i} f(z), \quad f \in H_{d}^{k}, z=\left(z_{1}, \ldots, z_{d}\right) \in \mathbb{B}_{d},
$$

and a reproducing kernel $k: \mathbb{B}_{d} \times \mathbb{B}_{d} \rightarrow \mathbb{C}$ with $k(\cdot, 0) \equiv 1$ is $\mathcal{U}$-invariant if for each unitary map $U: \mathbb{C}^{d} \rightarrow \mathbb{C}^{d}, k(U z, U w)=k(z, w)$ for all $z, w \in \mathbb{B}_{d}$. We find a class of reproducing kernel Hilbert spaces of analytic functions on $\mathbb{B}_{d}$, which includes many of the classic Hilbert spaces, such as the Dirichlet space and the weighted Bergman spaces, whose shift operators have the QW-property.

In order to state our main result, let us introduce two kinds of reproducing kernels. Fix a constant $v$ with $v>0$. Let $H_{d}^{k_{v}}$ be the Hilbert space on the unit ball defined by the following $\mathcal{U}$-invariant reproducing kernel:

$$
k_{v}(z, w)=\frac{1}{(1-\langle z, w\rangle)^{v}} .
$$

Note that when $v=1, H_{d}^{k_{v}}$ is the Arveson space $H_{d}^{2}$, which is studied by Arveson in $\mathrm{Arv}$. When $v=d, H_{d}^{k_{v}}$ is the usual Hardy space $H^{2}\left(\mathbb{B}_{d}\right)$, and when $v>d$, $H_{d}^{k_{v}}$ is the weighted Bergman space $L_{a}^{2}\left(\mathbb{B}_{d},\left(1-|z|^{2}\right)^{v-d-1} d V\right)$. Here $d V$ denotes the normalized volume measure on $\mathbb{B}_{d}$.

Recall that a reproducing kernel $k$ is called a complete Nevanlinna-Pick kernel if $k(\cdot, 0) \equiv 1$ and if $1-1 / k(z, w)$ is positive definite on $\mathbb{B}_{d} \times \mathbb{B}_{d}$. For the related 
details one can refer to $\mathrm{AMcC}$. Note that the Arveson space $H_{d}^{2}$ and the Dirichlet space $\mathcal{D}_{d}$ defined by the kernel

$$
k(z, w)=-\frac{1}{\langle z, w\rangle} \log (1-\langle z, w\rangle)
$$

have $\mathcal{U}$-invariant complete Nevanlinna-Pick kernels.

The following is our main result:

Theorem 1.1. If $k=k_{v}$ or $k$ is a $\mathcal{U}$-invariant complete Nevanlinna-Pick kernel, then the d-shift $\bar{S}$ on $H_{d}^{k}$ has the $Q W$-property.

We point out in passing that not on all Hilbert spaces $H_{1}^{k}$ with the kernels stated in Theorem 1.1 do the shift operators $S$ have the W-property. H. Hedenmalm and $\mathrm{K}$. Zhu ( $\mathrm{HZh}$ ) showed that the W-property can fail in certain weighted Bergman spaces $L_{a}^{2}\left(\mathbb{D},\left(1-|z|^{2}\right)^{v} d A\right)$ for some $v>1$, where $d A$ is the normalized area measure on $\mathbb{D}$. In addition, for a $\mathcal{U}$-invariant complete NP kernel $k(z, w)=\left(1-\frac{1}{17} z \bar{w}-\right.$ $\left.\frac{16}{17} z^{2} \bar{w}^{2}\right)^{-1}$ with $z, w \in \mathbb{D}, S$ has no W-property on the induced Hilbert space $H_{1}^{k}$ ([McCT] $)$. So the QW-property is quite different from the W-property.

\section{The PROOF OF THE MAIN RESUlt}

We first recall some properties concerning the $\mathcal{U}$-invariant reproducing analytic Hilbert space $H_{d}^{k}$. For more details one can refer to [GHX]. A routine verification shows that for a $\mathcal{U}$-invariant kernel $k$ on $\mathbb{B}_{d}$, there exists a unique power series $\sum_{n=0}^{\infty} a_{n} z^{n}$ on $\mathbb{D}$ with nonnegative coefficients $\left\{a_{k}\right\}$ satisfying

$$
k(z, w)=\sum_{n=0}^{\infty} a_{n}\langle z, w\rangle^{n}, \quad z, w \in \mathbb{B}_{d} .
$$

Here as usual $\langle z, w\rangle=\sum_{i=1}^{d} z_{i} \bar{w}_{i}$. Conversely, it is easy to check that if a power series $\sum_{n=0}^{\infty} a_{n} z^{n}$ has nonnegative coefficients, then $k$ defined by (2.1) defines a $\mathcal{U}$-invariant reproducing kernel on $\mathbb{B}_{d}$. In addition,

$$
\left\{\sqrt{a_{|\alpha|} \frac{|\alpha| !}{\alpha !}} z^{\alpha}: a_{|\alpha|} \neq 0\right\}
$$

forms a canonical orthonormal basis for the Hilbert space $H_{d}^{k}$. Here $\alpha=\left(\alpha_{1}, \ldots, \alpha_{d}\right)$ is a multi-index of nonnegative integers and

$$
|\alpha|=\alpha_{1}+\cdots+\alpha_{d}, \quad \alpha !=\alpha_{1} ! \cdots \alpha_{d} !, \quad z^{\alpha}=z_{1}^{\alpha_{1}} \cdots z_{d}^{\alpha_{d}} .
$$

We assume that $a_{n} \neq 0$ for $n \geq 0$ and $\sup _{n} \frac{a_{n}}{a_{n+1}}<\infty$, so that the $d$-shift $\bar{S}$ is bounded on $H_{d}^{k}$.

Let $k$ be as (2.1). Since $K(0,0)=1$, then we have

$$
\frac{1}{k(z, w)}=\sum_{n=0}^{\infty} c_{n}\langle z, w\rangle^{n}
$$

in some open neighborhood $U$ of zero. The multinomial formula gives that

$$
k(z, w)=\sum_{|\alpha|=0}^{\infty} a_{|\alpha|} \frac{|\alpha| !}{\alpha !} z^{\alpha} \bar{w}^{\alpha}, \quad \frac{1}{k(z, w)}=\sum_{|\beta|=0}^{\infty} c_{|\beta|} \frac{|\beta| !}{\beta !} z^{\beta} \bar{w}^{\beta}
$$


for $(z, w) \in U$. Note that the coefficients $\left\{a_{n}\right\}$ and $\left\{c_{n}\right\}$ satisfy

$$
c_{0} a_{0}=1, \quad \sum_{\beta \leq \alpha} c_{|\beta|} a_{|\alpha-\beta|} \frac{|\beta| !}{\beta !} \frac{|\alpha-\beta| !}{(\alpha-\beta) !}=0, \quad \alpha \neq 0,
$$

where and in what follows $\beta \leq \alpha$ means $\beta_{i} \leq \alpha_{i}$ for all $i=1, \ldots, d$, and

$$
\alpha-\beta=\left(\alpha_{1}-\beta_{1}, \ldots, \alpha_{d}-\beta_{d}\right) .
$$

Note that $a_{0}=1$, so $c_{0}=1$, and $c_{1}=-c_{0} a_{1} / a_{0}=-a_{1}<0$. In addition, $\left\{c_{n}\right\}$ is a sequence of real numbers.

For the $d$-shift $\bar{S}=\left(S_{1}, \ldots, S_{d}\right)$ on the Hilbert space $H_{d}^{k}$, we set

$$
Q_{n}=\sum_{|\beta| \leq n} c_{|\beta|} \frac{|\beta| !}{\beta !} \bar{S}^{\beta} \bar{S}^{* \beta}, \quad Q=\sum_{|\beta|=0}^{\infty} c_{|\beta|} \frac{|\beta| !}{\beta !} \bar{S}^{\beta} \bar{S}^{* \beta},
$$

where

$$
\bar{S}^{\beta}=S_{1}^{\beta_{1}} \cdots S_{d}^{\beta_{d}}, \quad \bar{S}^{* \beta}=S_{1}^{* \beta_{1}} \cdots S_{d}^{* \beta_{d}} .
$$

Proposition 2.1. For every polynomial $p$,

$$
Q(p)=p(0) .
$$

Hence, $Q$ can be extended continuously on $H_{d}^{k}$ as the evaluation functional at zero.

Proof. For every monomial $z^{\alpha}$, using the reproducing property of $k$, we get

$$
\begin{aligned}
S_{i}^{* \beta_{i}}\left[z^{\alpha}\right](z) & =\left\langle S_{i}^{* \beta_{i}} w^{\alpha}, k(\cdot, z)\right\rangle_{H_{d}^{k}}=\left\langle w^{\alpha}, w_{i}^{\beta_{i}} k(\cdot, z)\right\rangle_{H_{d}^{k}} \\
& =a_{\left|\alpha-\beta_{i} e_{i}\right|}\left\langle w^{\alpha}, w^{\alpha}\right\rangle_{H_{d}^{k}} \frac{\left|\alpha-\beta_{i} e_{i}\right| !}{\left(\alpha_{i}-\beta_{i}\right) !} z^{\alpha-\beta_{i} e_{i}}
\end{aligned}
$$

for $\beta_{i} \leq \alpha_{i}$, where $\alpha-\beta_{i} e_{i}=\left(\alpha_{1}, \ldots, \alpha_{i}-\beta_{i}, \ldots, \alpha_{d}\right)$. Then by (2.2),

$$
S_{i}^{* \beta_{i}} z^{\alpha}=\frac{\alpha_{i} !}{|\alpha| !} \frac{\left|\alpha-\beta_{i} e_{i}\right| !}{\left(\alpha_{i}-\beta_{i}\right) !} \frac{a_{\left|\alpha-\beta_{i} e_{i}\right|}}{a_{|\alpha|}} z^{\alpha-\beta_{i} e_{i}}
$$

for $\beta_{i} \leq \alpha_{i}$ and $S_{i}^{* \beta_{i}} z^{\alpha}=0$ for $\beta_{i}>\alpha_{i}$. Hence, the same arguments give that

$$
\bar{S}^{\beta} \bar{S}^{* \beta}\left(z^{\alpha}\right)=\left\{\begin{array}{cc}
\frac{\alpha !}{|\alpha| ! \frac{|\alpha-\beta| !}{(\alpha-\beta) !} \frac{a_{|\alpha-\beta|}}{a_{|\alpha|}} z^{\alpha},} & \beta \leq \alpha, \\
0, & \text { otherwise. }
\end{array}\right.
$$

It follows that $Q(1)=c_{0}=1$ and for every $\alpha \neq 0$, we have

$$
\begin{aligned}
Q\left(z^{\alpha}\right) & =\sum_{|\beta|=0}^{\infty} c_{|\beta|} \frac{|\beta| !}{\beta !} \bar{S}^{\beta} \bar{S}^{* \beta}\left(z^{\alpha}\right)=\sum_{\beta \leq \alpha} c_{|\beta|} \frac{|\beta| !}{\beta !} \bar{S}^{\beta} \bar{S}^{* \beta}\left(z^{\alpha}\right) \\
& =\sum_{\beta \leq \alpha} c_{|\beta|} \frac{|\beta| !}{\beta !} \frac{\alpha !}{|\alpha| !} \frac{|\alpha-\beta| !}{(\alpha-\beta) !} \frac{a_{|\alpha-\beta|}}{a_{|\alpha|}} z^{\alpha} \\
& =\frac{\alpha !}{|\alpha| ! a_{|\alpha|}} z^{\alpha} \sum_{\beta \leq \alpha} c_{|\beta|} a_{|\alpha-\beta|} \frac{|\beta| !}{\beta !} \frac{|\alpha-\beta| !}{(\alpha-\beta) !}=0
\end{aligned}
$$

by (2.3). Thus, for every polynomial $p$, we obtain $Q p=p(0)$, as desired.

The following lemma gives a sufficient condition for when $Q_{n}$ converges to $Q$ in SOT.

Lemma 2.2. If there exists a positive integer $N(\geq 2)$ such that $c_{i} \geq 0$ or $c_{i} \leq 0$ for all $i>N$, then $Q_{n} \rightarrow Q$ (SOT). 
Proof. Fix each $f(z)=\sum_{|\alpha|=0}^{\infty} b_{\alpha} z^{\alpha}$. It follows from (2.3) and (2.4) that we have

$$
\begin{aligned}
Q_{n} f-Q f & =\sum_{|\alpha|=1}^{\infty} b_{\alpha} \sum_{\beta \leq \alpha,|\beta| \leq n} c_{|\beta|} \frac{|\beta| !}{\beta !} \bar{S}^{\beta} \bar{S}^{* \beta}\left(z^{\alpha}\right) \\
& =\sum_{|\alpha|>n} b_{\alpha} \sum_{\beta \leq \alpha,|\beta| \leq n} c_{|\beta|} \frac{|\beta| !}{\beta !} \bar{S}^{\beta} \bar{S}^{* \beta}\left(z^{\alpha}\right) \\
& =\sum_{|\alpha|>n} b_{\alpha} z^{\alpha} \frac{\alpha !}{|\alpha| ! a_{|\alpha|}} \sum_{\beta \leq \alpha,|\beta| \leq n} c_{|\beta|} a_{|\alpha-\beta|} \frac{|\beta| !|\alpha-\beta| !}{\beta !} \frac{\mid \alpha-\beta) !}{(\alpha-\beta)}
\end{aligned}
$$

Hence we get

$$
\left\|Q_{n} f-Q f\right\|_{H_{d}^{k}}^{2}=\sum_{|\alpha|>n}\left|b_{\alpha}\right|^{2} \|\left. z^{\alpha}\right|_{H_{d}^{k}} ^{2}\left[\frac{\alpha !}{|\alpha| ! a_{|\alpha|}} \sum_{\beta \leq \alpha,|\beta| \leq n} c_{|\beta|} a_{|\alpha-\beta|} \frac{|\beta| !}{\beta !} \frac{|\alpha-\beta| !}{(\alpha-\beta) !}\right]^{2} .
$$

We claim that there is a constant $C>0$ such that

$$
\left|\frac{\alpha !}{|\alpha| ! a_{|\alpha|}} \sum_{\beta \leq \alpha,|\beta| \leq n} c_{|\beta|} a_{|\alpha-\beta|} \frac{|\beta| !}{\beta !} \frac{|\alpha-\beta| !}{(\alpha-\beta) !}\right| \leq C
$$

for $|\alpha| \geq N$ and $n \geq N$. Indeed, we first assume that when $n>N$ all $c_{n} \leq 0$. Note that $c_{0}=1, c_{1}<0$. It follows from (2.3) that

$$
\sum_{\beta \leq \alpha,|\beta| \leq n} c_{|\beta|} a_{|\alpha-\beta|} \frac{|\beta| !}{\beta !} \frac{|\alpha-\beta| !}{(\alpha-\beta) !} \geq 0
$$

for $|\alpha| \geq N$ and $n \geq N$, which gives

$$
\begin{aligned}
& \left|\frac{\alpha !}{|\alpha| ! a_{|\alpha|}} \sum_{\beta \leq \alpha,|\beta| \leq n} c_{|\beta|} a_{|\alpha-\beta|} \frac{|\beta| !}{\beta !} \frac{|\alpha-\beta| !}{(\alpha-\beta) !}\right| \\
& \leq \frac{\alpha !}{|\alpha| ! a_{|\alpha|}} \sum_{\beta \leq \alpha,|\beta| \leq N} c_{|\beta|} a_{|\alpha-\beta|} \frac{|\beta| !}{\beta !} \frac{|\alpha-\beta| !}{(\alpha-\beta) !} \\
& \quad=\sum_{\beta \leq \alpha,|\beta| \leq N} c_{|\beta|} \frac{a_{|\alpha-\beta|}}{a_{|\alpha|}} \frac{|\beta| !}{\beta !} \frac{|\alpha-\beta| !}{|\alpha| !} \frac{\alpha !}{(\alpha-\beta) !} \\
& \leq \max _{|\beta| \leq N} \frac{|\beta| !}{\beta !}\left|c_{|\beta|}\right| \sup _{\beta \leq \alpha,|\beta| \leq N} \frac{a_{|\alpha-\beta|}}{a_{|\alpha|}} \sup _{\beta \leq \alpha,|\beta| \leq N} \frac{\alpha !}{(\alpha-\beta) !}
\end{aligned}
$$

for $|\alpha| \geq N$ and $n \geq N$. Note that $\sup _{n} \frac{a_{n}}{a_{n+1}}<\infty$; thus it is easy to see that the claim follows. For the case when for $n>N$ all $c_{n} \geq 0$, the reasoning of the claim is similar.

It follows from (2.5) and (2.6) that

$$
\left\|Q_{n} f-Q f\right\|_{H_{d}^{k}}^{2} \leq C^{2} \sum_{|\alpha|>n}\left|b_{\alpha}\right|^{2}\left\|z^{\alpha}\right\|_{H_{d}^{k}}^{2} \rightarrow 0
$$

as $n \rightarrow \infty$ because of $\|f\|_{H_{d}^{k}}^{2}=\sum_{|\alpha|=0}^{\infty}\left|b_{\alpha}\right|^{2}\left\|z^{\alpha}\right\|_{H_{d}^{k}}^{2}<\infty$. This means that $Q_{n}$ converges to $Q$ in SOT. The proof is complete.

The following lemma is also useful in our proof. 
Lemma 2.3. Let $M$ be a closed subspace of a Hilbert space $H$ and $\bar{T}=\left(T_{1}, \ldots, T_{d}\right)$ be a d-tuple of commuting operators acting on $H$. Then

$$
M \ominus\left[P_{M} \bar{T} M^{\perp}\right]_{\bar{T}}=H \ominus\left[M^{\perp}\right]_{\bar{T}} .
$$

In particular, $\left[P_{M} \bar{T} M^{\perp}\right]_{\bar{T}}=M$ if and only if $\left[M^{\perp}\right]_{\bar{T}}=H$.

Proof. Suppose $f \in H \ominus\left[M^{\perp}\right]_{\bar{T}}$. Then

$$
f \perp T_{1}^{i_{1}} \cdots T_{d}^{i_{d}} M^{\perp}
$$

for all $i_{1}, \ldots, i_{d} \geq 0$. Obviously $f \in M$. Since

$$
\begin{aligned}
T_{1}^{i_{1}} \cdots T_{d}^{i_{d}} P_{M} T_{j} M^{\perp} & \subset T_{1}^{i_{1}} \cdots T_{d}^{i_{d}} T_{j} M^{\perp}-T_{1}^{i_{1}} \cdots T_{d}^{i_{d}} P_{M^{\perp}} T_{j} M^{\perp} \\
& \subset T_{1}^{i_{1}} \cdots T_{d}^{i_{d}} T_{j} M^{\perp}-T_{1}^{i_{1}} \cdots T_{d}^{i_{d}} M^{\perp},
\end{aligned}
$$

we have $f \perp T_{1}^{i_{1}} \cdots T_{d}^{i_{d}} P_{M} T_{j} M^{\perp}$ for all $i_{1}, \ldots, i_{d} \geq 0$ and $1 \leq j \leq d$. Hence $f \in M$ and $f \perp\left[P_{M} \bar{T} M^{\perp}\right]_{\bar{T}}$, that is, $f \in M \ominus\left[P_{M} \bar{T} M^{\perp}\right]_{\bar{T}}$.

For the converse, suppose $f \in M \ominus\left[P_{M} \bar{T} M^{\perp}\right]_{\bar{T}}$. Then

$$
f \perp T_{1}^{i_{1}} \cdots T_{d}^{i_{d}} P_{M} T_{j} M^{\perp}, \quad 1 \leq j \leq d, i_{1}, \ldots, i_{d} \geq 0 .
$$

Noting that

$$
\begin{aligned}
T_{1}^{i_{1}} \cdots T_{d}^{i_{d}} M^{\perp} & \subset T_{1}^{i_{1}} \cdots T_{d}^{i_{d}-1} P_{M} T_{d} M^{\perp}+T_{1}^{i_{1}} \cdots T_{d}^{i_{d}-1} P_{M^{\perp}} T_{d} M^{\perp} \\
& \subset T_{1}^{i_{1}} \cdots T_{d}^{i_{d}-1} P_{M} T_{d} M^{\perp}+T_{1}^{i_{1}} \cdots T_{d}^{i_{d}-1} M^{\perp}
\end{aligned}
$$

by induction, we have

$$
\begin{aligned}
T_{1}^{i_{1}} \cdots T_{d}^{i_{d}} M^{\perp} \subset & \sum_{i=1}^{i_{d}} T_{1}^{i_{1}} \cdots T_{d}^{i_{d}-i} P_{M} T_{d} M^{\perp} \\
& +\sum_{i=1}^{i_{d-1}} T_{1}^{i_{1}} \cdots T_{d-1}^{i_{d-1}-i} P_{M} T_{d-1} M^{\perp}+\cdots \\
& +\sum_{i=1}^{i_{1}} T_{1}^{i_{1}-i} P_{M} T_{1} M^{\perp}+M^{\perp} .
\end{aligned}
$$

From (2.7) we obtain that $f \perp T_{1}^{i_{1}} \cdots T_{d}^{i_{d}} M^{\perp}$ for every $i_{1}, \ldots, i_{d} \geq 0$. So $f \in$ $H \ominus\left[M^{\perp}\right]_{\bar{T}}$, and the proof is complete.

We are now ready to prove the main result.

Proof of Theorem 1.1. We first claim that if $Q_{n} \rightarrow Q$ (SOT or WOT), then $\bar{S}$ has the QW-property on $H_{d}^{k}$. In fact, if the claim is not true, then there is a nontrivial invariant subspace $M$ of $H_{d}^{k}$ for $\bar{S}$ such that $M \neq\left[P_{M} \bar{S} M^{\perp}\right]_{\bar{S}}$. Thus by Lemma2.3, there exists $f \in M$ with $f \neq 0$ such that $f \perp S_{1}^{i_{1}} \ldots S_{d}^{i_{d}} M^{\perp}$ for every $i_{1}, \ldots, i_{d} \geq 0$. It follows that $\bar{S}^{* \alpha} f \in M$ for all multi-indexes $\alpha$.

We write $f(z)=\sum_{|\alpha|=0}^{\infty} b_{\alpha} z^{\alpha}$ with some $b_{\alpha^{\prime}} \neq 0$ and let $h=\bar{S}^{* \alpha^{\prime}} f$. Then $h \in M$ and $\bar{S}^{* \alpha} h \in M$ for every multi-index $\alpha$, so $\bar{S}^{\alpha} \bar{S}^{* \alpha} h \in M$ for every $\alpha$. Note that $M$ is strongly (and weakly) closed. Hence by the condition and Proposition 2.1 .

$$
\sum_{|\beta|=0}^{\infty} c_{|\beta|} \frac{|\beta| !}{\beta !} \bar{S}^{\beta} \bar{S}^{* \beta} h=h(0)=b_{\alpha^{\prime}} \in M,
$$

which implies that $M=H_{d}^{k}$, a contradiction. So the claim holds. 
Now we only need to show that the two kinds of reproducing kernels in the theorem satisfy the condition of Lemma 2.2. In fact, if $k=k_{v}$ and $\frac{1}{k_{v}(z, w)}=$ $1+\sum_{n=1}^{\infty} c_{n}\langle z, w\rangle^{n}$, then

$$
c_{n}=(-1)^{n} \frac{v(v-1) \cdots(v-n+1)}{n !}, \quad n \geq 1 .
$$

It is easy to see that there exists a positive integer $N_{v}(\geq 2)$ such that $c_{n} \geq 0$ or $c_{n} \leq 0$ for all $n>N_{v}$. If $k$ is a complete Nevanlinna-Pick kernel and $\frac{\overline{1}}{k(z, w)}=$ $1+\sum_{n=1}^{\infty} c_{n}\langle z, w\rangle^{n}$, then [AMcC, Theorem 7.33] tells us that all $c_{n} \leq 0$ for $n \geq 1$. The proof is complete.

Remark 2.4. Let $H_{0}=\left\{f \in H_{d}^{k}: f(0)=0\right\}$ and $P_{0}$ be a projection onto $H_{0}$. Then

$$
P_{0}=I-Q=\sum_{|\beta|=1}^{\infty}\left(-c_{|\beta|}\right) \frac{|\beta| !}{\beta !} \bar{S}^{\beta} \bar{S}^{* \beta},
$$

where $I$ is the identity operator on $H_{d}^{k}$. If $k$ is a complete Nevanlinna-Pick kernel, then $c_{|\beta|} \leq 0$ for all $|\beta| \geq 1$. Therefore, the right side of the above equality is the infinite sum of positive operators and so it is easy to see that the sum converges in WOT (also see McCT, Lemma 1.4]).

The following corollary is a direct application of the main result. We call a $d$-tuple of operators $\bar{S}^{*}=\left(S_{1}^{*}, \ldots, S_{d}^{*}\right)$ the backward $d$-shift on $H_{d}^{k}$. Thus $M$ is an invariant subspace for the $d$-shift $\bar{S}$ if and only if $M^{\perp}$ is an invariant subspace for $\bar{S}^{*}$. So the following corollary tells us that two nontrivial invariant subspaces of $H_{d}^{k}$ for the backward $d$-shift $\bar{S}^{*}$ are never orthogonal, a result which generalizes Theorem 1 of [Gar] using different techniques.

Corollary 2.5. Let $M_{1}$ and $M_{2}$ be two invariant subspaces for $\bar{S}$ of $H_{d}^{k}$ with $k=k_{v}$ or $k$ is a $\mathcal{U}$-invariant complete Nevanlinna-Pick kernel. If $M_{1}^{\perp} \perp M_{2}^{\perp}$, then $M_{1}=H_{d}^{k}$ or $M_{2}=H_{d}^{k}$.

Proof. If $M_{1} \neq H_{d}^{k}$, then by Theorem 1.1 and Lemma 2.3. $\left[M_{1}^{\perp}\right]_{\bar{S}}=H_{d}^{k}$. Since the condition means that $M_{1}^{\perp} \subset M_{2}$, we have $M_{2}=H_{d}^{k}$, and the proof follows.

We finish this paper with the following natural question:

Question 2.6. Does the $d$-shift $\bar{S}$ on each reproducing analytic Hilbert space $H_{d}^{k}$ with $\mathcal{U}$-invariant kernel $k$ have the $\mathrm{QW}$-property?

For a general operator $T$ rather than the shift operator $S$, the question is not the case. For example, let $T=S^{2}$ and $M=H_{0}$, defined as in Remark 2.4. Then one easily sees that

$$
M \ominus\left[P_{M} S^{2} M^{\perp}\right]_{S^{2}}=\overline{\operatorname{span}}\left\{z, z^{3}, z^{5}, \cdots\right\} \neq\{0\} .
$$

\section{ACKNOWLEDGEMENTS}

The author is deeply grateful to his advisor, Prof. Kunyu Guo, for his help and encouragement during the author's doctoral studies. He also thanks Prof. Tao Yu and Prof. Huiming $\mathrm{Xu}$ for many valuable discussions related to this work and the referee for many helpful suggestions which make this paper more readable. 


\section{REFERENCES}

[ARS] A. Aleman, S. Richter, C. Sundberg, Beurling's theorem for the Bergman space, Acta Math. 177 (1996), 275-310. MR 1440934 (98a:46034)

[AMcC] J. Ager, J. McCarthy, Pick interpolation and Hilbert function spaces, Graduate Studies in Mathematics, 44, American Mathematical Society, Providence, Rhode Island, 2002. MR1882259 (2003b:47001)

[Arv] W. Arveson, Subalgebras of $C^{*}$-algebras III: multivariable operator theory, Acta Math. 181 (1998), 159-228. MR 1668582 (2000e:47013)

[Beu] A. Beurling, On two problems concerning linear transformations in Hilbert space, Acta Math. 81 (1949), 239-255. MR0027954 (10:381e)

[Gar] S. Garcia, The backward shift on Dirichlet-type spaces, Proc. Amer. Math. Soc. 133 (10) (2005), 3047-3056. MR2159784 (2006e:30038)

[GHX] K. Guo, J. Hu, X. Xu, Toeplitz algebras, subnormal tuples and rigidity on reproducing $C\left[z_{1}, \cdots, z_{d}\right]$-modules, J. Funct. Anal. 210 (2004), 214-247. MR2052120 (2005a:47007)

[Hed] H. Hedenmalm, An invariant subspace of the Bergman space having the codimension two property, J. Reine Angew. Math. 443 (1993), 1-9. MR1241125 (94k:30092)

[HZh] H. Hedenmalm, K. Zhu, On the failure of optimal factorization for certain weighted Bergman spaces, Complex Variables Theory Appl. 19 (3) (1992), 165-176. MR.1284108 (95f:30064)

[Izu] K. J. Izuchi, K. H. Izuchi, Y. Izuchi, Quasi-wandering subspaces in the Bergman space, Integr. Equ. Oper. Theory 67 (2010), 151-161. MR.2650767

[McCT] S. McCullough, T. Trent, Invariant subspaces and Nevanlinna-Pick kernels, J. Funct. Anal. 178 (2000), 226-249. MR.1800795 (2002b:47006)

[Ric] S. Richter, Invariant subspaces of the Dirichlet shift, J. Reine Angew. Math. 386 (1988), 205-220. MR936999 (89e:47048)

Department of Mathematics, Zhejiang Normal University, Jinhua, 321004, People's RePublic OF China

E-mail address: ychen227@gmail.com 